\title{
ESTUDIO HISTOLÓGICO Y MORFOLÓGICO DEL DESARROLLO EMBRIONARIO DEL PEZ CAPITÁN DE LA SABANA (Eremophilus mutisii)
}

\section{HISTOLOGICAL AND MORPHOLOGICAL STUDY OF THE EMBRIONARY DEVELOPMENT OF THE CAPITAN DE LA SABANA (Eremophilus mutisii)}

\begin{abstract}
Erika Moncaleano Gómez ${ }^{1}$, Carlos Sánchez ${ }^{2}$, Camilo Prieto Mojica ${ }^{3}$
${ }^{1}$ Zootecnista, M.Sc. Universidad de Ciencias Aplicadas y Ambientales U.D.C.A, Bogotá, Colombia, e-mail: erikamarcelamg@ gmail.com, (D)https://orcid.org/0000-0001-6807-2877; ${ }^{2}$ Zootecnista. Universidad de Ciencias Aplicadas y Ambientales U.D.C.A Bogotá, Colombia, e-mail: carlsanchez@udca.edu.co, iDhttps://orcid.org/0000-0002-7470-679X; ${ }^{3} Z$ Zootecnista, M.Sc. Ph.D., Profesor, Facultad de Ciencias Pecuarias, Programa de Zootecnia. Universidad de Ciencias Aplicadas y Ambientales U.D.C.A, Bogotá, Colombia, e-mail: camprieto@udca.edu.co, iDhttps://orcid.org/0000-0002-4987-3596
\end{abstract}

Rev. U.D.C.A. Act. \& Div. Cient. 21(2):479-489, Julio-Diciembre, 2018 https://doi.org/10.31910/rudca.v21.n2.2018.1073

Artículo de acceso abierto publicado por Revista U.D.C.A Actualidad \& Divulgación Científica bajo una licencia Creative Commons CC BY-NC 4.0

\section{RESUMEN}

Eremophilus mutisii, pez catalogado en estado como vulnerable, que por su importancia sociocultural y ecológica genera retos investigativos, uno de ellos, conocer sus características histológicas del desarrollo embrionario, desde la fecundación hasta la eclosión. Para ello, se obtuvieron embriones de individuos adultos y maduros sexualmente, capturados en la represa del Sisga, ubicada en el municipio de Chocontá, Cundinamarca, inducidos sexualmente con extracto pituitario de carpa. Los ovocitos y el semen fueron obtenidos por presión abdominal 24 horas después de la última dosis, a $14 \pm 1^{\circ} \mathrm{C}$. La toma de muestras, se realizó cada 60 minutos, durante las primeras doce horas post-fertilización (HPF); a partir de aquí y hasta la eclosión, las muestras fueron fijadas en formol Bufferado al 4\%, cada 6 horas y sometidas a rutina de histología. Los resultados mostraron que los óvulos fertilizados presentaron forma esférica, color verde claro y diámetro promedio de $1270 \mu \mathrm{m}$. El primer clivaje, se observó a las 2HPF; la blastulación inició a las 9HPF; los movimientos epibólicos y la gastrulación, se observaron desde la 12HPF hasta la 30HPF, con el cierre del blastoporo. A las 60HPF, se evidenció el desprendimiento de la cola del saco vitelino y a las $66 \mathrm{HPF}$, se dio inicio a los primeros movimientos autónomos. Finalmente, a las 72HPF inició la eclosión, mostrando las larvas ausencia de pigmentación y evidenciándose un tracto digestivo rudimentario, sin abertura bucal y anal. Se observó la presencia de abundante saco vitelino, con movimientos verticales continuos de la larva.

Palabras clave: desarrollo embrionario, capitán de la sabana, embriogénesis, Siluriformes, Trichomycteridae.

\section{ABSTRACT}

Eremophilus mutisii, due to its vulnerable condition and its potential for fish farming, generates research challenges; one of them is to know the embryonic changes of the E. mutisii based on morphological and histological features from fertilization to hatching. The embryos were obtained from adult and sexually mature individuals of the Capitán de la Sabana (Eremophilus mutisii), sexually induced with carp pituitary extract. Oocytes and semen were obtained by abdominal pressure 24 hours after the last dose at $14 \pm 1^{\circ} \mathrm{C}$. Sampling was performed every 60 minutes for the first twelve hours' post-fertilization (HPF); from here on at hatching, the samples were fixed every 6 hours. The embryos were fixed in $4 \%$ formalin for observing the morphological features and for the histological studies. Results revealed that the fertilized eggs had spherical shape, light green color and average diameter of $1270 \mu \mathrm{m}$. The first cleavage at $2 \mathrm{HPF}$ was observed, the blastulation began at 9 HPF. Epibolic movements and gastrulation were observed from $12 \mathrm{HPF}$ to $30 \mathrm{HPF}$ with blastopore closure. At $60 \mathrm{HPF}$ the yolk sac tail detachment was evident and at $66 \mathrm{HPF}$ the first autonomous movements began. Fi- 
nally, at $72 \mathrm{HPF}$, the hatching began, showing absence of pigmentation and evidencing a rudimentary digestive tract without oral and anal opening. It was observed the presence of abundant yolk sac with continuous vertical movements of the larva.

Keywords: embryonic development, capitan de la Sabana, embryogenesis, Siluriformes, Trichomycteridae.

\section{INTRODUCCIÓN}

El capitán de la sabana (Eremophilus mutisii) es una especie íctica endémica del altiplano Cundiboyacense, emblemática dentro de la cultura andina. Pertenece a la familia Trichomycteridae; su distribución geográfica, se localiza en la cuenca del río Magdalena, según Maldonado-Ocampo et al. (2008) y en la sub-cuenca del Altiplano Cundí-boyacense, entre los 2.500 y los $3.100 \mathrm{~m}$ s.n.m., donde la temperatura ambiental no sobrepasa los $18-20^{\circ} \mathrm{C}$ (Mojica et al. 2012).

Los principales estudios realizados en el E. mutisii son ecológicos (Flórez \& Sarmiento, 1989), biométricos (Cala, 1985) y reproductivos (Rodríguez \& Rosado, 1993), por lo cual, existe un vacío en el conocimiento respecto a las primeras etapas de vida de la especie, indispensables para un posible aprovechamiento piscícola. Según Feiden et al. (2006) es importante el estudio del desarrollo de embriones y larvas, con dos propósitos principales: conservación y producción en cautiverio, justificando el estudio de las características morfofisiológicas del desarrollo embrionario, que proporciona información sobre su ciclo vital, permitiendo un mejor manejo de la especie.

El desarrollo embrionario es utilizado para estudios ontogénicos, modelos experimentales y evaluaciones, acerca de las cualidades y los efectos de sustancias tóxicas en el ambiente acuático (Ninhaus et al. 2006). Estudios en bagre yaque (Leiarius marmoratus) (Zapata-Berruecos et al. 2008) y en bagre rayado (Psedoplatystoma sp.) (Marques et al. 2016) muestran que entre especies de la misma familia existen marcadas diferencias, en cuanto a la cronología del desarrollo embrionario, lo cual, hace necesario realizar trabajos, para conocer la embriología de cada especie, en particular.

El pez E. mutisii ha sido catalogado como el bagre de agua dulce con mayores probabilidades de piscicultura en zonas frías alto andinas (Rosado \& González, 2007). La disminución poblacional de E. mutisii o, incluso, su desaparición en algunos cuerpos de agua naturales, ocasionó que, en el 2012, fuera catalogada como especie vulnerable en el Libro Rojo de los Peces (Mojica et al. 2012).

Al respecto, González-M. et al. (2014), encontraron altos niveles de Plomo en el músculo, en el $93 \%$ de los ejemplares de capitán de la sabana muestreados, sin observar efectos fisiológicos o patológicos derivados de este hallazgo y recomiendan mantener una vigilancia sobre las implicaciones que pueda tener este hallazgo al futuro, en el estado sanitario de la especie.

Mojica et al. (2012) sugieren medidas de conservación, propuestas para la protección de hábitat, educación ambiental y el desarrollo de programas de reproducción; no obstante, se requieren estudios de la etapa de incubación y de larvicultura de esta especie, que permitan, a futuro, la obtención de alevinos de esta especie.

Por tanto, el objetivo del presente trabajo fue describir morfológica e histológicamente, el desarrollo embrionario de $E$. mutisii, desde la fecundación hasta la eclosión de las larvas.

\section{MATERIALES Y MÉTODOS}

Los embriones, se obtuvieron de individuos adultos y maduros sexualmente de E. mutisii, capturados en la represa del Sisga, ubicada en el municipio de Chocontá, Cundinamarca $\left(5^{\circ} 05^{\prime} 17^{\prime \prime} N 73^{\circ} 43^{\prime} 18^{\prime \prime} \mathrm{W}\right)$. Estos peces fueron transportados al laboratorio, ubicado en las instalaciones de la Unidad Académica El Remanso, de la Universidad de Ciencias Aplicadas y Ambientales U.D.C.A, sede Bogotá, situada a 2.600m.s.n.m. y temperaturas que oscilan entre 9 y $20^{\circ} \mathrm{C}$ (IDEAM, 2016).

Una vez adaptados los animales al cautiverio, se procedió a realizar el protocolo de inducción hormonal, con extracto de hipófisis de carpa (EPC). El número de animales utilizados en el protocolo de reproducción fue 2 hembras y 3 machos.

El protocolo utilizado fue el propuesto por González et al. (2017), donde la dosis total para las hembras fue de 5,5mg de $E P C / k g$, de peso de la hembra, con un intervalo de 12 horas; las modificaciones planteadas frente a este protocolo fueron dadas en una mayor dosis para las hembras y cambio en los intervalos de dosis, puesto que la primera dosis fue de $0,25 \mathrm{mg} / \mathrm{kg}$ de peso de la hembra; la segunda dosis, $0,5 \mathrm{mg} /$ $\mathrm{kg}$ de peso de la hembra, 28 horas después de la primera dosis y, la tercera, fue $5 \mathrm{mg} / \mathrm{kg}$ de peso de la hembra, 12 horas después de la segunda dosis. A los machos, se les hizo una sola aplicación de $4 \mathrm{mg} / \mathrm{kg}$ de peso de cada macho, a la misma hora de la tercera dosis de la hembra.

Los ovocitos y el semen fueron obtenidos por presión abdominal, 24 horas después de la última dosis, a $15^{\circ} \pm 0,5^{\circ} \mathrm{C}$. El proceso de fertilización, se realizó mezclando el esperma y los ovocitos; luego, se adicionó a la mezcla agua, para activar los espermatozoides y permitir la hidratación de los huevos. Una vez fecundados, se trasladaron a incubadoras tipo bandeja, en tanques de plástico de 250L, con sistema de 
recirculación, filtro biológico y temperatura controlada, en un promedio de $15 \pm 0,6^{\circ} \mathrm{C}$.

Para determinar la duración del desarrollo embrionario, se consideró, como momento cero, la hora de activación de los gametos y como hora final, el momento en que el $90 \%$ de los embriones de una muestra aleatoria, alcanzó la eclosión (Jhonston et al. 1995).

La toma de muestras, se realizó cada 60 minutos, durante las primeras doce horas post-fertilización (HPF); a partir de aquí y hasta la eclosión, las muestras fueron fijadas cada 6 horas. En cada muestra, se tomó, al menos, 10 unidades, fijadas en formalina bufferada al $4 \%$, para su posterior análisis descriptivo.

En total, se observaron 23 muestras, en las 3 bandejas de incubación. Con estas, se procedió a la descripción del desarrollo embrionario, utilizando un microscopio óptico Nikon Eclipse Ci con cámara Nikon DS-Fi 1 y pantalla digital Nikon Sight.

Consecutivamente, las muestras fueron fijadas en formol bufferado $10 \%$, para rutina histológica, sometidas a un proceso de deshidratación e inclusión en parafina (MERCK, punto de fusión $52-54^{\circ} \mathrm{C}$ ) (Agarwal, 1996; Jaramillo et al. 2009); se realizaron cortes transversales de $5 \mu \mathrm{m}$ de espesor, en un microtómo rotatorio, los cuales, se tiñeron con la técnica de Mayer y Harris, hematoxilina-Eosina (Agarwal, 1996). Seguidamente, estas se analizaron y se fotografiaron, para su descripción histológica, en un microscopio Nikon eclipse E-600 y cámara digital Nikon DXM 1200.

\section{RESULTADOS Y DISCUSIÓN}

El desove, se realizó a las 24 horas, posteriores a la aplicación de la dosis definitiva a los reproductores, bajo las condiciones de cautiverio, en las que la temperatura de mantenimiento fue de $15,2 \pm 0,5^{\circ} \mathrm{C}$, que corresponde a 364 horas grado. En condiciones semejantes, González et al. (2017) encontraron que el periodo de respuesta fue a las 290 a 345 horas grado. El procedimiento completo, desde la primera dosis hormonal y el desove, tuvo una duración de 64 horas.

Los ovocitos de E. mutisii, al momento del desove, son esféricos, con diámetro promedio de 1.270 micras; presentan abundante vitelo, de color verde claro (Figura 1a). Al momento de la hidratación, se visualizó la presencia de una doble membrana. En esta etapa, el ovocito aumenta de tamaño, alcanzando 1.484 micras de diámetro, la primera membrana y 2.037 micras de diámetro, la membrana más externa (Figura 1b, 1c y 1d). De la hora cero a la hora uno
HPF ocurrió la diferenciación del polo animal, por la presencia de los pronúcleos y el polo vegetal, por la concentración de vitelo (Figura 1h).

Los huevos de los peces, se pueden clasificar como telolecitos, debido a que acumulan el vitelo en el polo vegetal y presentan una división parcial o meroblástica durante los primeros estadios del desarrollo (Ninhaus et al. 2006). Los huevos de $E$. mutisii, se ajustan a esta clasificación y, por lo tanto, presentan divisiones meroblásticas o parciales, limitadas al polo animal, como se describe también para el pez $P$. grosskopfii (Valbuena-Villareal et al. 2012).

Las observaciones del desarrollo embrionario en E. mutisii muestran que el tiempo hasta la eclosión es más largo que en otras especies de siluridos de aguas cálidas (Marques et al. 2008; Faustino et al. 2007; Honji et al. 2012).

La presencia de doble membrana, se debe a una adaptación morfológica que brinda protección al embrión en aguas con alta turbidez (Cardoso et al. 1995; Pereira et al. 2006). Esta característica, se presenta en ovocitos de peces siluriformes, como el Pseudoplatystoma sp. (Díaz-Olarte et al. 2010; Marques et al. 2008), coincidiendo con nuestros hallazgos. Adicionalmente, tiene función de protección del embrión y adhesión a diversos materiales, para evitar daños en los mismos.

Respecto al análisis histológico, se aprecia externamente una estructura delgada, ligeramente basofílica, soportada sobre una lámina eosinofílica, la cual, corresponde a la membrana vitelina, separada de las otras estructuras del huevo, por un espacio claro, que corresponde a la cápsula.

Periodo de segmentación. La blastomeración es de tipo meroblastico; el primer clivaje ocurrió a las 2HPF (Figura 1d, $1 \mathrm{e})$, dividiendo el blastodisco verticalmente, para formar dos células de igual tamaño. La segunda división ocurrió perpendicular a la primera, a las tres HPF, originando cuatro blastómeros (Figura 1f,1g).

A nivel histológico, se empieza a observar la agrupación del material particulado eosinofílico, que conforma el vitelo hacia uno de los polos, denominado polo vegetal; este material es el más abundante y ocupa el $80 \%$ del huevo. Hacia el otro extremo, se empieza a depositar el material eosinofilo tenue homogéneo, haciendo evidente el polo animal; este material ocupa el 20\% del embrión (Figura 1h).

A las 4HPF, la división fue doble y paralela al primer eje del clivaje (Figura 2a, 2b, 2c, 2d). El cuarto clivaje ocurrió a las 5HPF, originando 16 blastómeros (Figura 2e, 2f). 


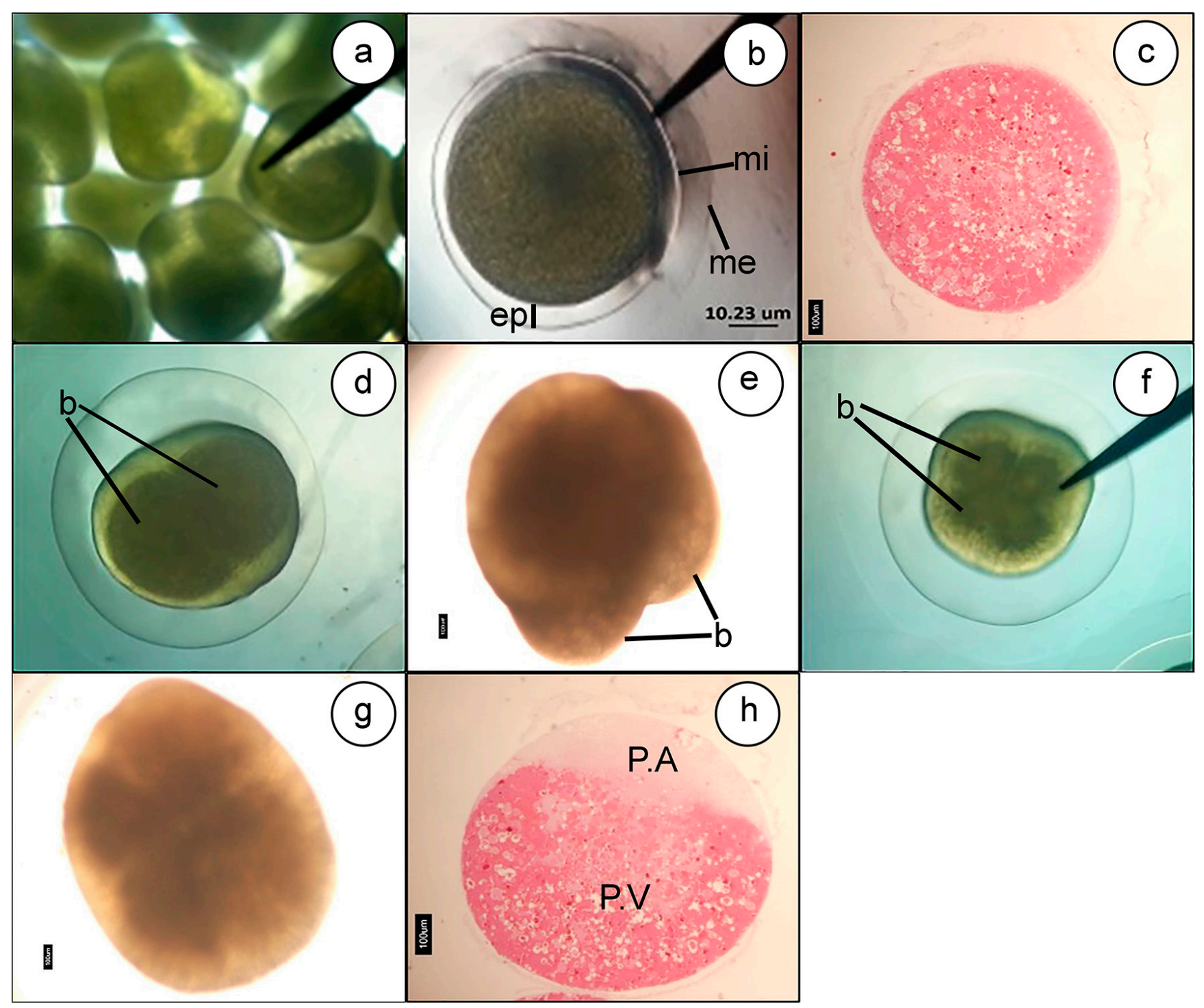

Figura 1. Ovocitos y clivaje de Eremophilus mutisii. a. Huevos maduros (4x); b. Huevo recién fertilizado (4x), 0 horas postfertilización (HPF); c. Huevo en tinción H\&E (4x), 0 horas postfertilización (HPF); d y e. Primeras divisiones celulares (4x), 2 horas postfertilización (HPF); f y g. 4 divisiones celulares (4x), 3 horas postfertilización (HPF); h. Diferenciación del polo vegetal y animal (4x), 2 horas postfertilización (HPF), tinción de H\&E. mi) membrana interna; me) membrana externa; ep) espacio perivitelino; c) Corion; P.A) Polo animal; P.V) Polo vegetal; b) Blastómero. 


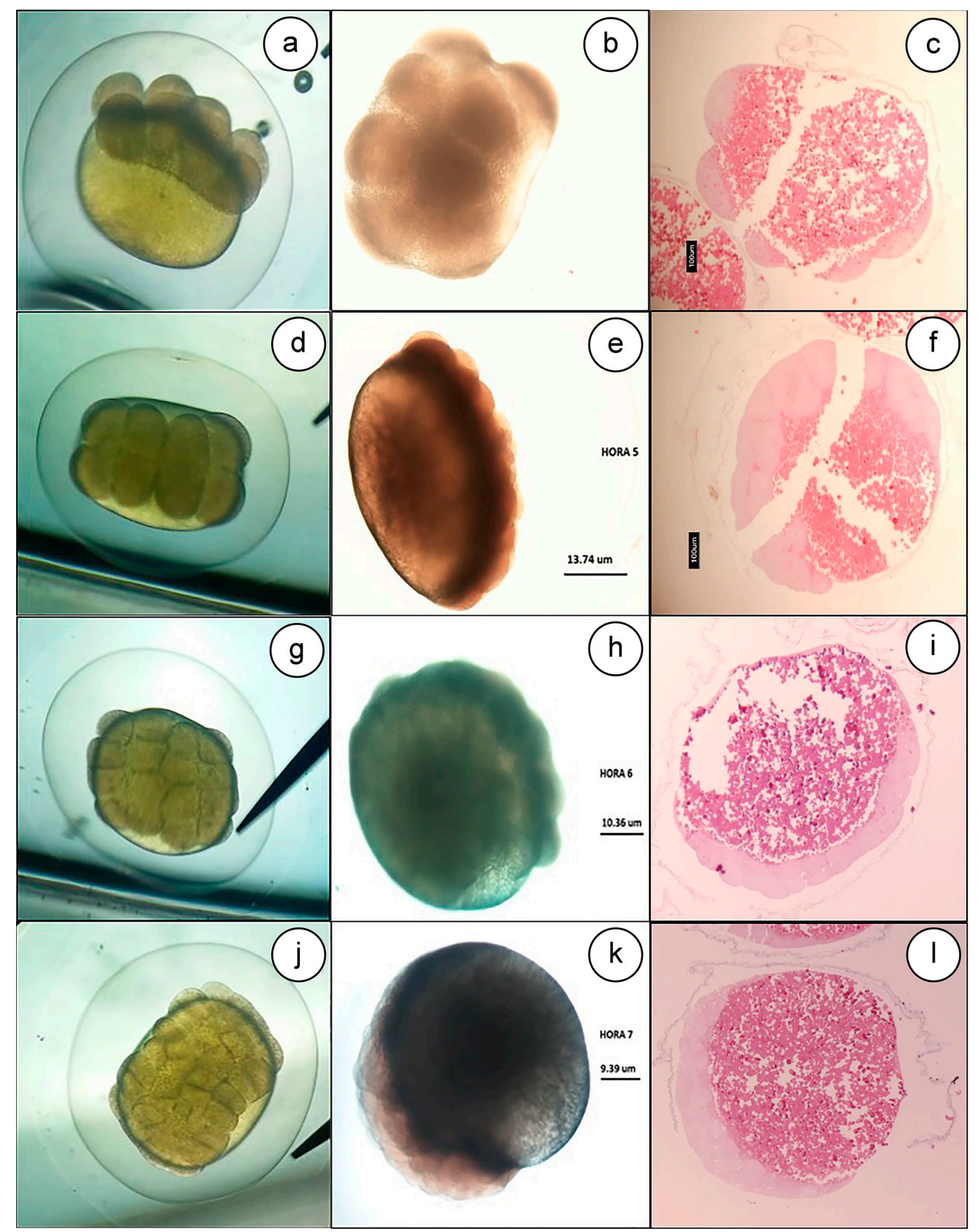

Figura 2. Clivaje de Eremophilus mutisii. a, b y c. 8 divisiones celulares (4x), 4 horas postfertilización (HPF); d, e y f. 16 divisiones celulares (4x), 5 horas postfertilización (HPF); G, H y I) 32 células (4x), 6-7 horas postfertilización (HPF); j, k y l. 64 células (4x), 7-8 horas postfertilización (HPF); c, f, i y l. Tinción en H\&E. t) Tabiques o Septos. 
Los primeros clivajes observados en la especie estudiada, siguen el mismo patrón de segmentación que los reportados por otros silúridos, tales como Rhamdia quelen (Pereira et al. 2006), P. grosskopfii (Valbuena-Villareal et al. 2012), P. corruscans (Marques et al. 2008), Zungaru jau (Marques et al. 2017) y en el hibrido de $P$. corruscans x P. fasciatum (Faustino et al. 2007).

Adicionalmente, se empieza a hacer evidente la segmentación, división embrionaria o clivaje del manto embriónico, limitada en esta especie a la zona del blastodisco (segmentación meroblástica discoidal). Esta segmentación, se hizo evidente por la presencia de 8 estructuras de gran tamaño, con aspecto de cúpula o domo, formando un montículo en la superficie del polo animal, las cuales, corresponden a las células (blastómeros), que se forman en la fase de clivaje en esta hora. Estas células contienen un material eosinofílico tenue homogéneo y, a pesar de presentar una ligera separación entre ellas por un tabique o septo, todas se encuentran interconectadas (Figura 2c).

Del quinto al sexto clivaje, se originan 32 blastómeros (Figura $2 \mathrm{~g}, 2 \mathrm{~h}, 2 \mathrm{i}$ ) y 64 blastómeros (Figura 2j, 2k, 2l), lo cual, dificulta su visualización y, por lo tanto, su conteo, en virtud de tamaño y disposición de las células.

El tiempo transcurrido desde la fertilización hasta llegar al quinto clivaje, 32 células y sexto clivajes, 64 células, fluctuó entre 6 y 8HPF, en E. mutisii. Para otros siluridos, como el Pseudoplatystoma sp., el tiempo para este estado embrionario fluctúa entre 1 y 2HPF (Díaz-Olarte et al. 2010). Cabe resaltar que la diferencia de temperatura del agua en que viven estas especies contribuye a tiempos de desarrollo más cortos.

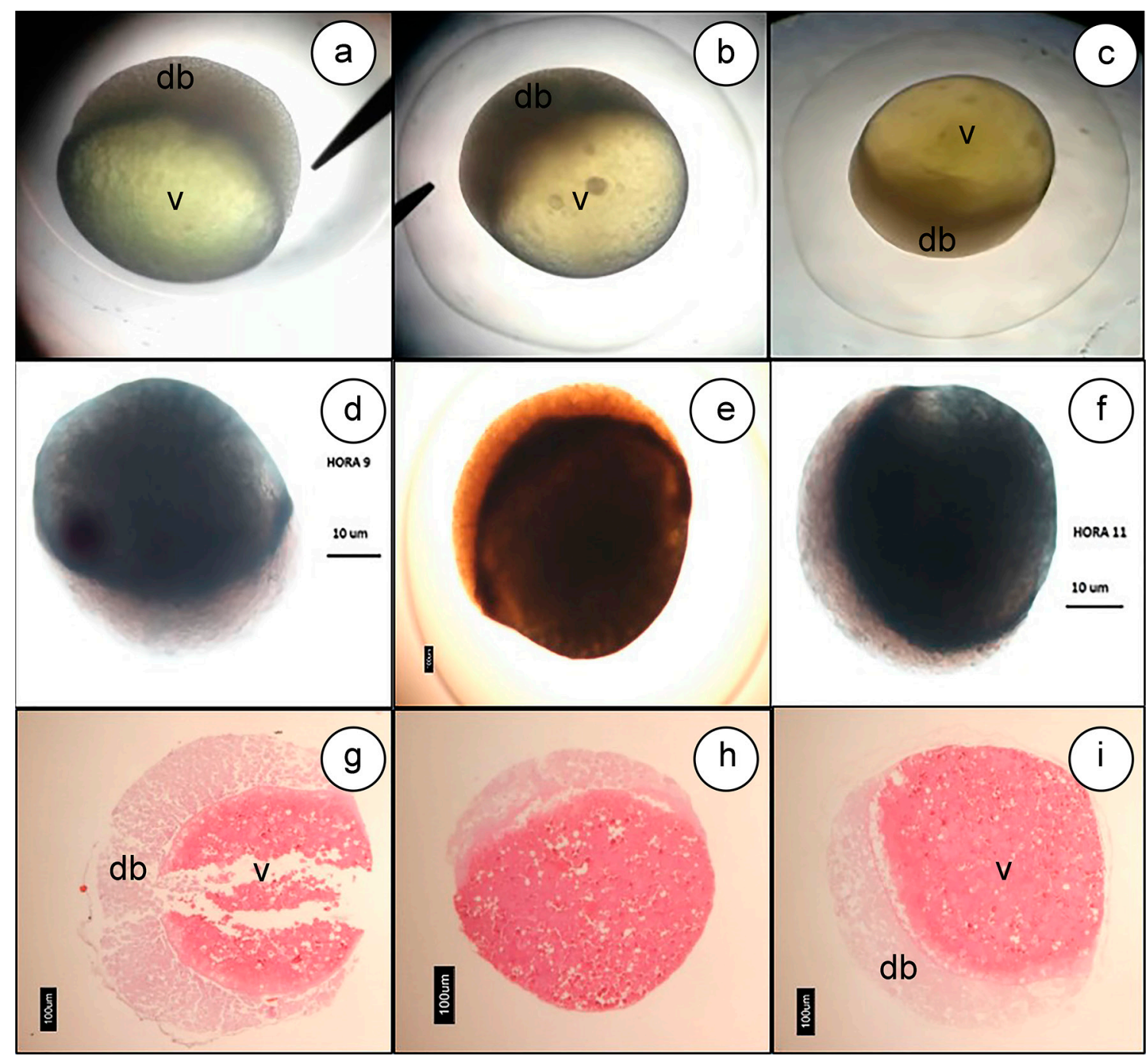

Figura 3. Blastulación Eremophilus mutisii: Elevación del blastodisco 9HPF (4x) (a, d y g.); elevación del blastodisco 10HPF (4X) (b, e y h); inicio del descenso del blastodisco sobre el polo vegetal 11,12HPF (4x) (c, f e i.); Hematoxilina -Eosina ( $g$, h, e i.). db) Blastodisco; v) Vitelo. 
También, se comenzaron a observar algunas vacuolas en el citoplasma de estas células y los pronúcleos basofílicos se hacen un poco más visibles y toman forma de línea o barra. Los tabiques o septos delgados que separan las células son mucho más prolongados, pudiéndose observar casi una completa separación entre las células, aunque todavía se mantienen interconectadas por pequeñas áreas (Figura 2c, 2f, 2i, 2l).

Periodo de blastulación. La blástula, se observó como una capa celular que empieza a envolver el vitelo, que recibe la denominación de blastodisco, dándole el embrión una forma ovalada. Este periodo, se caracterizó por 3 etapas: inicial, de elevación y de achatamiento, esta última marca el final de la blastulación y el comienzo de la gastrulación (Figura 3).

Los hallazgos microscópicos consistieron en la presencia de la membrana vitelina, delgada, ligeramente basofílica, soportada sobre una lámina eosinofílica y rodeando concéntricamente el huevo. El polo animal contiene células con un material eosinofílico tenue homogéneo, con núcleos basófilos bien visibles, ubicados en el centro de la célula, la mitad en forma de barra y la otra redondeada. Adicionalmente, se observó que, en esta hora, las células son más redondas y se observa una separación de las mismas, que deja espacios translucidos entre ellas, a veces, dando la apariencia que las células se están encogiendo. También, se observaron algunas de las vacuolas citoplasmáticas reportadas anteriormente (Figura 3g, 3h y 3i).

El paso de blástula a gástrula, conocido como el proceso de epibolia, en E. mutisii alcanzó el 50\%, luego, llegó al 80$95 \%$, hasta llegar al cierre del blastoporo, donde finalmente el epiblasto forma el ectodermo y el hipoblasto crea la notocorda y el mesodermo (Arratia et al. 2003).

Adicionalmente, los cambios más significativos, en esta fase, correspondieron a poder observar vacuolas citoplasmáticas y los pronúcleos basofílicos de manera más visible, algunos redondeados y otros en forma de barras, dando la apariencia de estar en proceso de mitosis. Cerca al vitelo, se observa una capa de células alargadas con núcleos ovalados, ligeramente basofílicos, que sirven de soporte al polo animal, conteniendo hasta 2 y 3 núcleos de diferentes tamaños; estas células, se denominan sincitiales vitelinas, debido a que se caracterizan por tener varios núcleos, resultante de la fusión de varias células (García, 1973) y están presentes en el vitelo.

Las vacuolas contenidas en las células del blastóforo son pleomórficas y dorsal al blastóforo; se comienzan a apreciar una capa de células columnares, las cuales, corresponden a células mesenquimales, que tienen un potencial proliferativo, capaz de auto renovarse, formando células idénticas a las células de origen y la capacidad de generar uno o más tipos celulares, que desempeñan funciones especializadas en el organismo (Mayani, 2003; Flores et al. 2006).

Periodo de gastrulación. Las células blastodérmicas y periblásticas comienzan a envolver el vitelo, mediante movimientos epibólicos (Figura 4a, 4g, 4j). La epibolia llegó al 50\%, a la hora 18 post fertilización (Figura 4d, 4e, 4f); en la hora 24 post fertilización, el porcentaje de epibolia llega al 70-80\% (Figura $4 \mathrm{~g}, 4 \mathrm{~h}, 4 \mathrm{i}$ ); en la última etapa, se observa el cierre del blastoporo, haciéndose más evidente la diferenciación del eje cráneo-caudal, hora 30 post fertilización (Figura 4j, $4 \mathrm{k}, 4 \mathrm{l})$.

Periodo de organogénesis. En esta etapa, se observa la diferenciación céfalo-caudal de simetría bilateral y el comienzo de la neurolación, a las 42HPF (Figura 5a, 5b, 5c). A las $48 \mathrm{HPF}$, se observa la vesícula óptica, vesícula ótica y la formación de las primeras somitas (Figura 5d, 5e, 5f). Este proceso en el Pseudoplatystoma sp., se observa entre la 8-16HPF (Díaz-Olarte et al 2010); en el P. grosskopfii, a las 7HPF (Valbuena-Villareal et al. 2012) y en Zungaru jau, a las 6HPF (Marques et al. 2017).

Respecto al contenido del embrión, el polo animal ocupó un $30 \%$ y se caracterizó por presentar diferenciación céfalo-caudal; la parte más grande del polo animal corresponde a la región cefálica y la más pequeña, a la caudal. Todo el blastoporo estuvo poblado por células pequeñas y medianas, con núcleos redondeados basofílicos y citoplasmas vacuolados, casi en un $90 \%$. El borde del vitelo presentó una capa de células sincitiales vitelinas, más delgadas con células aplanadas y núcleos alargados, semejante a un epitelio escamoso simple.

Se apreció una capa de células columnares, las cuales, corresponden a células mesenquimales del tubo neural (Figura $5 \mathrm{~g})$. Al lado de esta estructura, se evidenciaron 10 estructuras triangulares o piramidales, compuestas de células mesenquimales, que corresponden a somitas (Figura 5h).

Hacia la 60HPF, se evidenció el desprendimiento de la cola del saco vitelino (Figura 5i, 5j); a las 66HPF (Figura 5k, 5l), se observaron los primeros movimientos autónomos, diferenciando el corazón y el inicio de la circulación periférica, con movimientos cada vez más fuertes, acompañados de numerosas contracciones caudales y marcado deterioro de la membrana.

El vitelo ocupó el $60 \%$ del embrión, conteniendo material particulado eosinofílico pleomórfico, el cual, hacia la zona de la cabeza y cola, comenzó a perder la eosinofilia y se volvió más vacuolado, dando la apariencia de vacuolas semejantes al tejido adiposo. El blastodermo cubrió en forma de semiluna el vitelo, que estuvo poblado por células pequeñas y me- 


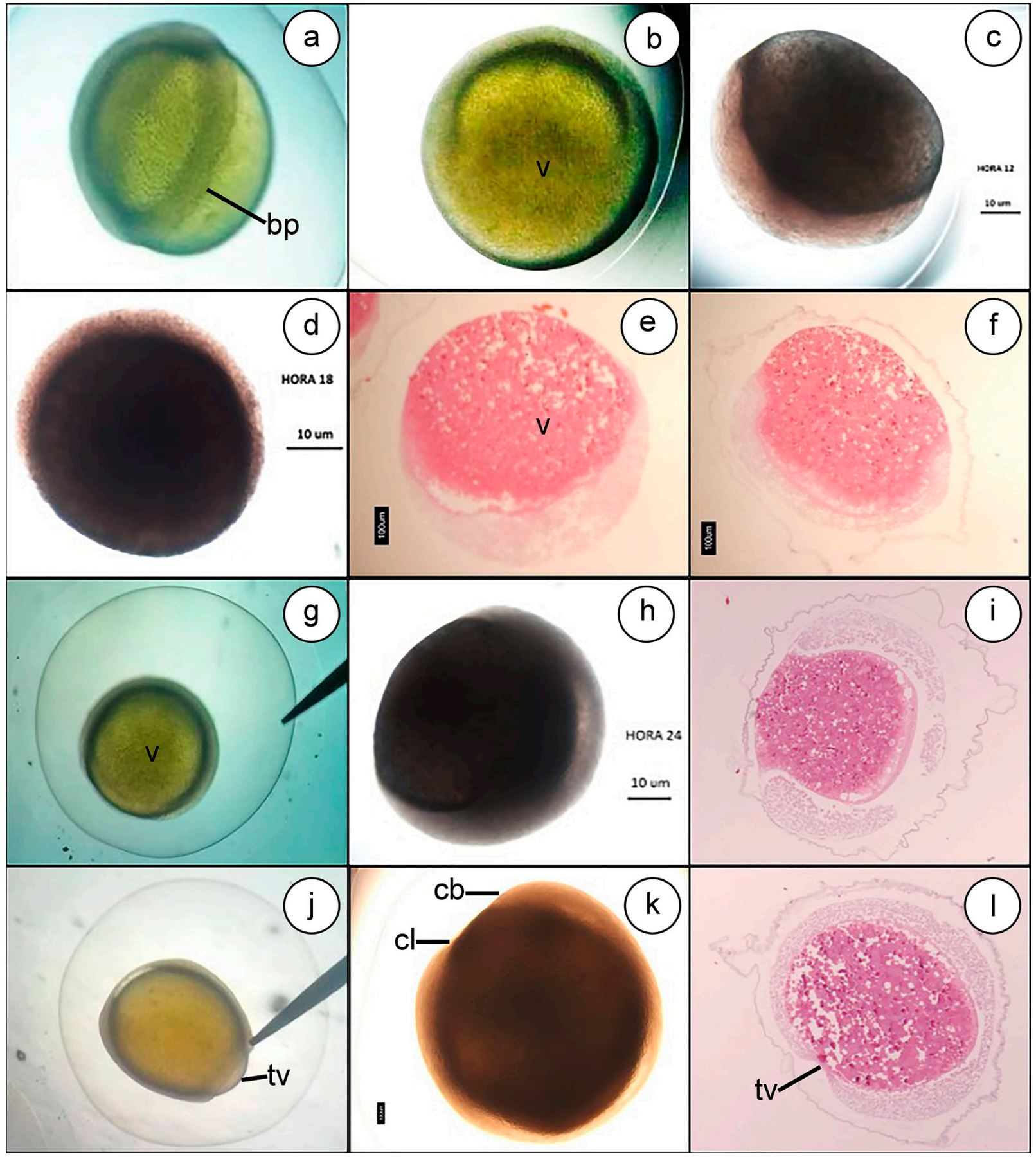

Figura 4. Gastrulación Eremophilus mutisii: Inicio de la epibolia, 12HPF (a, b y c.); epibolia al 50\%, 18HPF (d, e y f.); epibolia al $70-80 \%$, 24HPF (g, h e i.); cierre del blastoporo, 30HPF (j, k y l). bp) Borde del periblasto; v) Vitelo; tv) Tapón del vitelo; cb) Cabeza; cl) Cola.

dianas, con núcleos redondeados basofílicos y citoplasmas vacuolados, casi en un $80 \%$. El borde del vitelo presentó una capa de células aplanadas, con núcleos alargados basófilos. En esta hora, se pudo observar el notocordio, similar al tejido del cordón espinal en animales adultos, con las somitas triangulares, debajo de este. Hacia la región craneal, se observó una estructura circular rodeada por una membrana, aparentemente la vesícula óptica, que presentaba múltiples células en apoptosis. 


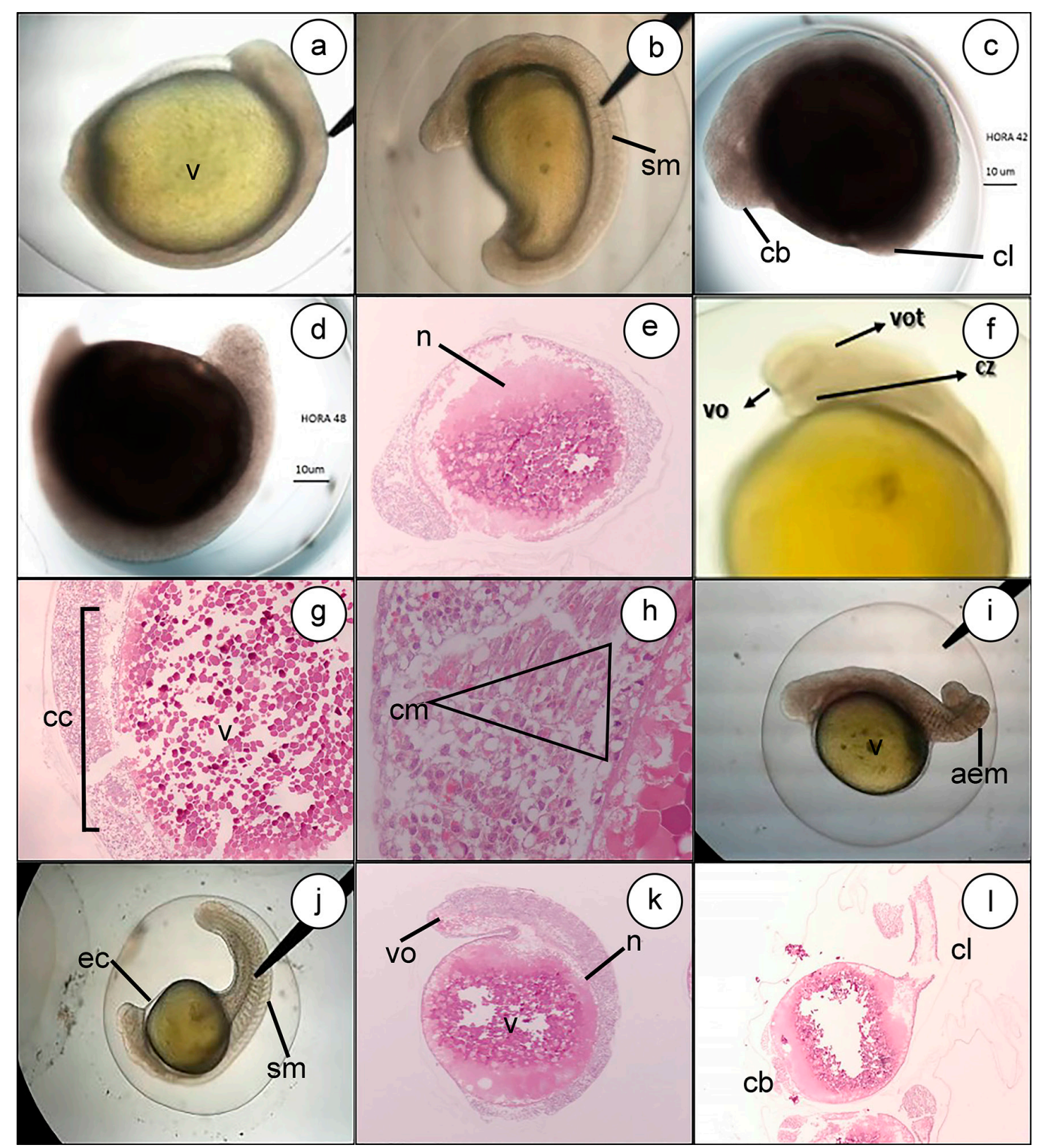

Figura 5. Organogénesis Eremophilus mutisii. 42HPF (a, b y c) 4X. Diferenciación céfalo caudal de simetría bilateral, formación de las primeras somitas y el comienzo de la neurolación. Se observa 48HPF (d, e y f.) 4X. Vesícula óptica, ótica y corazón. (G) Capa de células columnares, las cuales, corresponden a células mesenquimales del tubo neural. H\&E (H) Estructuras triangulares, compuestas de células mesenquimales. H\&E 60HPF (i y j), se evidenció desprendimiento de la cola del saco vitelino. 66HPF (k y l) H\&E. V) vitelo; sm) Somitas; cb) Cabeza; cl) Cola; n) Notocordio; vo) Vesícula óptica; vot) Vesícula ótica; cz) Corazón; cc) Capa de Células Columnares; Cm) Células Mesenquimales; aem) Aleta embrionaria; ec) Ectodermo; sm) Somitas; vo) Vesícula Óptica; n) Notocordio; v) Vitelo; cb) Cabeza; cl) Cola.

La duración de la organogénesis, se mostró entre las 42HPF y 66HPF. Respecto a otros silúridos es mucho mayor, como es el caso del Pseudoplatystoma corruscans, que se observó entre las 7-14HPF (Cardoso et al. 1995). En especies, como P. grosskopfii, se observa entre las 7-11HPF (Valbuena-Villareal et al. 2012).
Eclosión. La eclosión, se inició a las 72HPF (Figura 6), correspondiente a 1.096 horas grado, con una temperatura promedio de $15,2 \pm 0,6^{\circ} \mathrm{C}$. Las larvas mostraban ausencia de pigmentación y un tracto digestivo rudimentario, debido una estructura tubular con un lumen; esta estructura no tenía abertura bucal y anal. Se observó la presencia de abundante saco vitelino con movimientos verticales continuos de 
la larva. A las seis horas post eclosión, el volumen del saco vitelino es de $1,1 \mu \mathrm{m}^{3}$ y la longitud total es de $3,7 \mathrm{~mm}$, mayor a las larvas de Pimelodus maculatus, que eclosionan a las 14 h 30 min post-fertilización, a $25,5 \pm 0,3^{\circ} \mathrm{C}$, midiendo 2,56 \pm 0,13mm (Luz et al. 2001).

El vitelo, se observó homogéneo y continuo, perdiendo más la eosinofilia y presentando múltiples vacuolas, semejantes a las del tejido adiposo.

E. mutisii tiene un desarrollo embrionario más lento que otras especies, como Pseudoplatystoma corruscans (Marques et al. 2008), Steindachneridion parahybae (Honji et al. 2012) o el hibrido entre Pseudoplatystoma Corruscans y Pseudoplatystoma fasciatum (Faustino et al. 2007) y ello, se debe, principalmente, a que la temperatura de incubación fue más baja que las especies descritas anteriormente y la razón radica en que los teleósteos son vertebrados ectotérmicos.

Los datos actuales, por lo tanto, mostraron que el estudio del desarrollo embrionario es esencial para un mejor conocimiento de algunas características biológicas de una especie.
Estos resultados contribuyen a la recuperación de esta especie amenazada, al proporcionar herramientas biológicas, que permiten predecir y resolver problemas futuros, aportando, así, a la conservación y uso de la especie, para estudios posteriores de crecimiento en cautiverio, como alternativa de producción en aguas frías.

Agradecimientos: Los autores expresan su agradecimiento al laboratorio de histopatología animal de la Universidad Nacional de Colombia, sede Bogotá, por su colaboración en el procesamiento de muestras histológicas y registro fotográfico de las mismas. Conflicto de intereses: El manuscrito fue preparado y revisado con la participación de todos los autores, quienes declaramos que no existe conflicto de intereses que ponga en riesgo la validez de los resultados presentados. Financiación: Este estudio fue realizado bajo la cofinanciación del Departamento Administrativo de Ciencia, Tecnología e Innovación (Colciencias) y la Universidad de Ciencias Aplicadas y Ambientales U.D.C.A, en el proyecto: "Producción en cautiverio del capitán de la sabana, Eremophilus mutisii con fines de conservación".

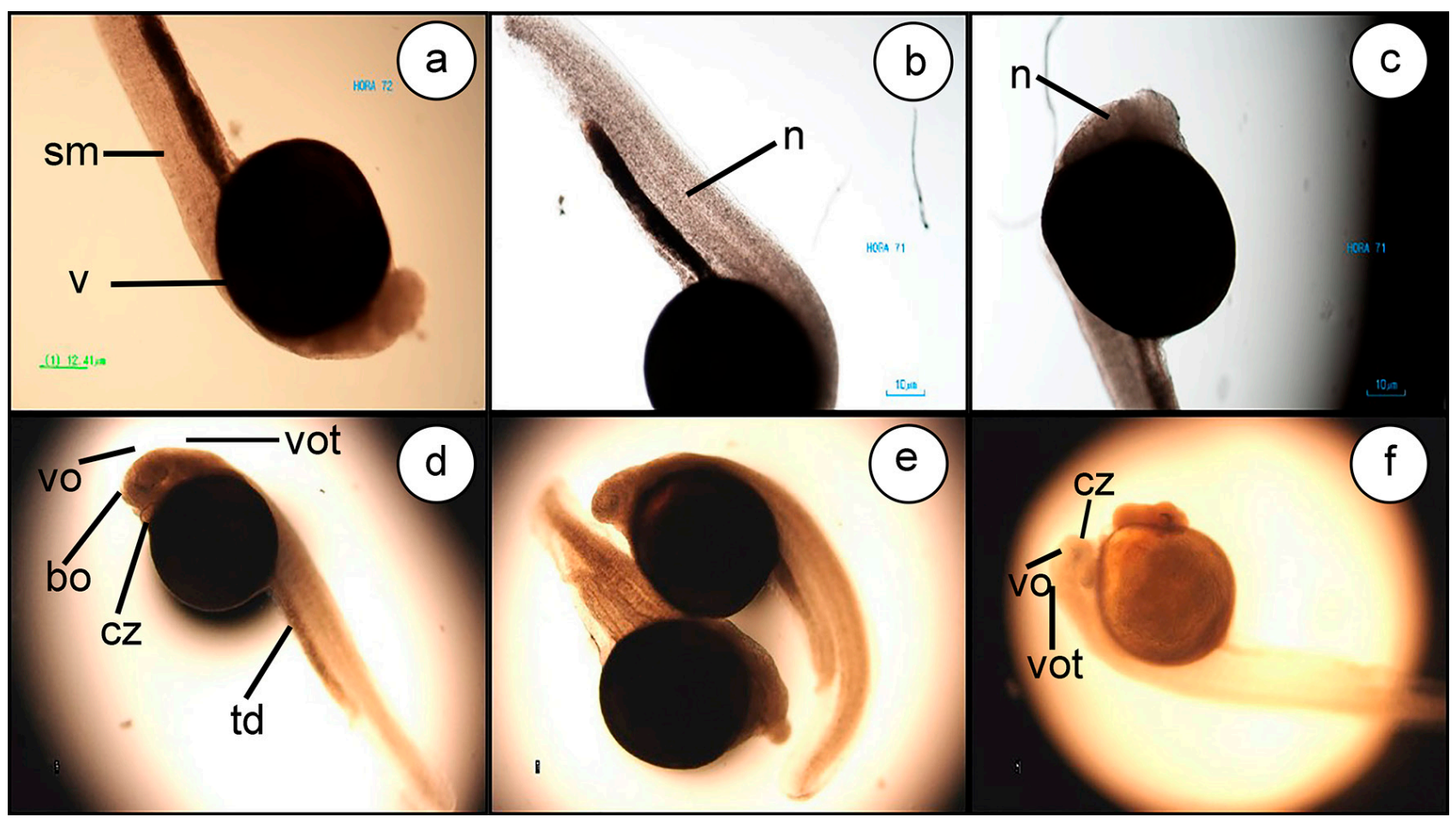

Figura 6. Eclosión de Eremophilus mutisii 72 HPF. a, b y c. 4X; d, e y f. 20X. v) Vitelo; sm) Somitas; n) Notocordio; vo) Vesícula Óptica; vot) Vesícula Ótica; bo) Bulbo olfatório; cz) Corazón; td) Tracto digestivo rudimentario. 


\section{REFERENCIAS}

1. AGARWAL, N.K. 1996. Fish Reproduction. APH Publishing Corporation Efficient offset printers, New Delhi, Indian. 120p.

2. ARRATIA, G.; KAPOOR, B.; DIOGO, R.; CHARDON, M. 2003. Catfishes. Vol 2. Editorial. Edenbridge Ltd. p.639-668.

3. CALA, P. 1985. Cambios histomorfológicos en los testes del capitán, Eremophilus mutisii, (Trichomycteridae, Siluriformes), durante el ciclo reproductivo anual en el sistema del río Bogotá en Colombia. Caldasia (Colombia). 14(68-70):659-677.

4. CARDOSO, E.L.; ALVES, M.S.D.; FERREIRA, R.M.A.; GODINHO, H.P. 1995. Embryogenesis of the neotropical freshwater Siluriforme Pseudoplatystoma coruscans. Aquat. Living Resour. (Francia). 8(4):343346. https://doi.org/10.1051/alr:1995037

5. DÍAZ-OLARTE, J.; MARCIALES-CARO, L.; CRISTANCHO, V.; CRUZ-CASALLAS, P. 2010. Comparación del desarrollo embrionario de Piaractus brachypomus (Serrasalmidae) y Pseudoplatystoma sp. (Pimelodidae). Internal J. Morphology (Chile). 28(4):1193-1204. https://doi.org/10.4067/S071795022010000400033

6. FAUSTINO, F.; NAKAGHI, L.S.O.; MARQUES, C.; MAKINO, L.; SENHORINI, J.A. 2007. Fertilizacao e desenvolvimento embrionário: morfometria e análise estereomicroscópica dos ovos dos híbridos de surubins (pintado, Pseudoplatystoma corruscans $\times$ cachara, Pseudoplatystoma fasciatum). Acta Sci. Biol. Sciences (Brasil). 29:49-55. https://doi. org/10.4025/actascibiolsci.v29i1.126

7. FEIDEN, A.; HAYASHI, C.; BOSCOLO, W.R.; REIDEL, A. 2006. Desenvolvimento de larvas de Steindachneridion sp. em diferentes condições de refúgio e luminosidade. Pesquisa Agropecuária Brasilia. 41:133-137. https://doi.org/10.1590/ S0100-204X2006000100018

8. FLORES, E.; MONTESINOS, J.; MAYANI, H. 2006. Células troncales mesenquimales: historia, biologia y aplicación clinica. Rev. de Invest. Clin. (Mexico). 58(5):498-511.

9. FLÓREZ, F.; SARMIENTO, N. 1989. Observaciones ecológicas sobre el pez capitán, Eremophilus mutisii Humbolt 1805 (Pisces: Trichomycteridae) en los De- partamentos de Cundinamarca y Boyacá - Colombia. Acta Biológica Colombiana.1(5):99-115.

10. GARCÍA, F. 1973. Fundamentos de biología. Minerva Books, Ed.Texas, (USA). 526p.

11. GONZÁLEZ-M., J.; LANDINES, M.; BORBÓN, J.; CORREAL, M.; SÁNCHEZ, C.; RODRÍGUEZ, L. 2014. Evaluación de algunos marcadores de exposición a contaminantes en tres especies de bagres colombianos (Pisces: Siluriformes). Biota Colombiana. 15(supl 1):40-51.

12. GONZÁLEZ, J.; ZAPATA, B.; ROSADO, R. 2017. Desarrollo embrionario del capitan de la sabana, Eremophilus mutisii (Pisces: Trichomycteridae). Revista de Medicina Veterinaria (Colombia). 35:137-147. http:// dx.doi.org/10.19052/mv.4396

13. HONJI, R.M.; TOLUSSI, C.E.; MELLO, P.H.; CANEPPELE, D.; RENATA, G.M. 2012. Embryonic development and larval stages of Steindachneridion parahybae (Siluriformes: Pimelodidae) - implications for the conservation and rearing of this endangered Neotropical species. Neotropical Ichthyology. 10(2):313-327. http://dx.doi.org/10.1590/S167962252012005000009

14. IDEAM. 2016. Instituto de Hidrología, Meteorología y Estudios Ambientales. Disponible desde Internet en: http://www.ideam.gov.co/ (con acceso el 25/11/2016).

15. JARAMILLO, J.; GOMEZ-RAMIREZ, E.; CALDAS, M.L.; RODRIGUEZ, D.; HURTADO, H. 2009. Histology and Morphometry of dorsal root ganglia and their neurons in a fish of indeterminate growth the white Cachama (Piaractus brachypomus). Actual. Biol. 31:43-52.

16. JHONSTON, I.A.; VERA, L.; VIERA, A. 1995. Temperature and myogenesis in embryos of the Atlantic herring Clupea harengus. J. Experimental Biology (Reino Unido). 198:1389-1403.

17. LUZ, R.K.; REYNALTE-TATAJE, D.A.; FERREIRA, A.A.; ZANIBONI-FILHO, E. 2001. Desenvolvimento embrionário e estágios larvais do mandi-amarelo Pimelodus maculatus. Boletim do Instituto de Pesca. 27:49-55.

18. MALDONADO-OCAMPO, J.; VARI, R.; USMA, J. 2008. Checklist of the Freshwater Fish of Colombia. Biota Colombiana. 9(2):143-237. 
19. MARQUIES, C.; FAUSTINO, F.; BERTOLUCCI, B.; PAES, M.D.C.F.; DA SILVA, R.C.; SATIKO, L.O.N. 2017. Embryonic development in Zungaro jahu. Zygote. 25(1):17-31. https://doi.org/10.1017/ S0967199416000277

20. MARQUES, C.; SATIKO, L.O.N.; FAUSTINO, F.; GANECO, L.N.; SENHORINI, J.A. 2016. Observation of the embryonic development in Pseudoplatystoma coruscans (Siluriformes: Pimelodidae) under light and scanning electron microscopy. Zygote 16:333342. http://doi.org/10.1017/S0967199408004838

21. MARQUES, C.; OKADA-NAKAGHI, L.S.; FAUSTINO, F.; NAKAGHI-GANECO, L.; SENHORINI, J.A. 2008. Observation of the embryonic development in Pseudoplatystoma corruscans (Siluriformes: Pimelodidae) under light and scanning electron microscopy. Zygote. 16:333-342. https://doi.org/10.1017/ S0967199408004838

22. MAYANI, H. 2003. A glance into somatic stem cell biology: basic principles, new concepts, and clinical relevance. Arch. Med. Res. (Mexico). 34(1):3-15. https:// doi.org/10.1016/S0188-4409(02)00450-2

23. MOJICA, J.; USMA, J.; ÁLVAREZ-LEON, R.; LASSO, C.A. (Eds). 2012. Libro rojo de peces dulceacuícolas de Colombia 2012. Instituto de Investigación de Recursos Biológicos Alexander von Humboldt, Instituto de Ciencias Naturales de la Universidad Nacional de Colombia, WWF Colombia y Universidad de Manizales. Bogotá, D.C., Colombia. 319p.

24. NINHAUS, A.; FORESTI, F.; AZEVEDO, A. 2006. Structural and ultrastructural analysis of embrionic develoment of Prochilodus lineatus (Valenciennes, 1836) (Characiforme; Prochilodontidae). Zigote. (England). 4(3):217-229. http://doi.org/10.1017/ S096719940600373X
25. PEREIRA, C.R.; BARCELLOS, L.J.G.; KREUTZ, L.C.; QUEVEDO, R.M.; RITTER, F.; SILVA, L. 2006. Embryonic and larval develoment of jundiá (Rhamdia quelen, Quoy and Gaimard, 1824, Pisces, Teleostei), a South American catfish. Brazilian J. Biology. 66(4):1057-1063. https://doi.org/10.1590/S151969842006000600013

26. RODRÍGUEZ, A.; ROSADO, R. 1993. Ensayos de reproducción inducida en capitán de la sabana Eremophilus mutisii, Humboldt, 1805. Boletín Red Regional de Acuicultura CIID-Colciencias. 7(3):10-13.

27. ROSADO, R.; GONZÁLEZ, J. 2007. Compilación bibliográfica y análisis del estado actual de la investigación sobre capitán de la sabana, Eremophilus mutisii. Dahlia (Colombia). 9:43-51.

28. VALBUENA-VILLARREAL， R.; ZAPATA-BERRUECOS, B.; DAVID-RUALES, C.; CRUZ-CASALLAS, P. 2012. Desarrollo Embrionario del Capaz Pimelodus grosskopfii (Steindachner, 1879). Int. J. Morphol. (Chile). 30(1):150-156. http://dx.doi.org/10.4067/S071795022012000100027

29. ZAPATA-BERRUECOS, B.E.; RAMÍREZ-MERLANO, J.A.; OTERO-PATERNINA, A.M.; MEDINA-ROBLES, V.M.; VELASCO-SANTAMARÍA, Y.M.; CRUZ-CASALLAS, P.E. 2008. Descripción preliminar del desarrollo embrionario de yaque Leiarius marmoratus. IV Congreso Colombiano de Acuicultura. Rev. Colomb. Cienc. Pecu. 21:513.

Recibido: Febrero 20 de 2018

Aceptado: Octubre 31 de 2018

Cómo citar:

Moncaleano Gómez, E.; Sánchez, C.; Prieto Mojica, C. 2018. Estudio histológico y morfológico del desarrollo embrionario del pez capitán de la sabana (Eremophilus mutisii). Rev. U.D.C.A Act. \& Div. Cient. 21(2):479-489. https://doi.org/10.31910/ rudca.v21.n2.2018.1073 